\title{
LIFE+ Traisen: Der lange Weg zum neuen Fluss
}

\author{
Thomas Kaufmann · Gerd Frik · Roland Schmalfuß · Gertrud Haidvogl · Jürgen Eberstaller · Helmut Wimmer · \\ Mathias Jungwirth
}

Online publiziert: 4. April 2018

(c) Der/die Autor(en) 2018.

Zusammenfassung Der Artikel „LIFE+ Traisen: Der lange Weg zum neuen Fluss" beschreibt die Geschichte der Entwicklungen und notwendigen Maßnahmen von den ersten Ideen bis über den Projektstart hinaus.

Das Vorhaben „Neue Traisen“ ist eines der größten ökologischen Flussbauprojekte in Österreich bzw. Europa. Ziel war die Herstellung einer Flusslandschaft in Niederösterreich mit einem $9,5 \mathrm{~km}$ langen, neuen Flusslauf und dessen Mündung bei der Donau. Obwohl der Bau selbst effektiv und effizient in nur 3 Jahren ausgeführt werden konnte, dauerten Konzeption, Planung, Finanzierung und Genehmigungen der Behörden über 15 Jahre. Das besonders gut gelungene aber herausfordernde Projekt konnte nur durch Zusammenarbeit vieler Beteiligter und durch eine gut organisierte Projektleitung realisiert werden.

\section{LIFE + Traisen: The long way to the new river}

Abstract The article "LIFE+ Traisen: The Long Way to the New River" describes the history of developments and

DI Dr. T. Kaufmann ( $\triangle)$

Büro freiwasser,

Währingerstraße 135/18, 1180 Wien,

Österreich

kaufmann@freiwasser.at

Mag. G. Frik • DI Dr. R. Schmalfuß . DI H. Wimmer

VERBUND Hydro Power GmbH Europaplatz 2, 1150 Wien, Österreich

Mag. Dr. G. Haidvogl •

Univ.-Prof. Dr. M. Jungwirth

Institut für Hydrobiologie und

Gewässermanagement (IHG),

Universität für Bodenkultur Wien,

Gregor-Mendel-Straße 33, 1180 Wien,

Österreich

DI Dr. J. Eberstaller

ezb - TB Eberstaller GmbH,

Schopenhauerstraße 82/12, 1180 Wien, Österreich necessary measures from initial ideas to beyond the start of the project.

The project "Neue Traisen" is one of the largest ecological river engineering projects in Austria and in Europe. The aim was to create a river landscape in Lower Austria with a $9.5 \mathrm{~km}$ long, new river and its mouth at the Danube. Although construction itself could be accomplished effectively and efficiently in just 3 years, design, planning, financing and permits of the authorities took over 15 years. The successful but challenging project could only be realized through cooperation and well-organized project management.

\section{Was ist die neue Traisen?}

Das Augebiet zwischen Traismauer und Zwentendorf war historisch von der Donau und deren Nebenarmen geprägt und bis zum Bau des Donaukraftwerks Altenwörth nicht von der Traisen durchflossen. Der Mündungsbereich des Traisen-Flusses lag bei der heutigen Marina Traismauer. Wegen der Aufhöhung des Donauspiegels im Stauraum musste der Traisen-Fluss verlängert und in das Unterwasser des Kraftwerkes geleitet werden. Diese Umleitung wurde den damaligen wasserbaulichen Standards entsprechend durch ein geradliniges, $7,5 \mathrm{~km}$ langes, völlig unstrukturiertes Gerinne realisiert, das zudem noch mehrere Sohlrampen und mit Blocksteinen gesicherte Ufer besaß.

Die „neue Traisen“ ist ein ökologisches Flussbauprojekt, das mit seinen Maßnahmen auf die Herstellung einer völlig neuen Flusslandschaft zielt. Der etwa $10 \mathrm{~km}$ lange Abschnitt des neu angelegten Flusses ist in das bestehende Augebiet eingebettet. Das Projekt wurde von VERBUND Hydro Power GmbH (VHP; bis 2010 VERBUND - Austrian Hydro Power AG) mit Förderung durch das LIFE+ Programm der Europäischen Union und nach dem Umweltförderungsgesetz (UFG) sowie mit finanzieller Unterstützung durch Projektpartner umgesetzt. Die bauliche Fertigstellung des neuen Flusses erfolgte Ende 2016 nach drei Jahren Bauzeit. Die Vorbereitungszeit dauerte wesentlich länger.

Das Vorhaben „neue Traisen“ ist kein klassisches Flussrückbauprojekt, weil kein Flusslauf rückgebaut wurde. Im Gegenteil, im Augebiet musste erst ein neuer Flusslauf geschaffen werden. Für die ökologisch funktionelle Ausformung des Flussbetts waren große Mengen Aushub nötig. Die Verwertung des entnommenen Kieses trug in untergeordnetem Ausmaß auch zur Finanzierung des Vorhabens bei.

Dieser Artikel beschreibt jene Phasen und Maßnahmen, die bei der Vorbereitung des Vorhabens erforderlich waren.

\section{Das Gewässerbetreuungs- konzept Traisen 1995 bis 1999}

Gewässerbetreuungskonzepte (GBKs) sind übergeordnete schutzwasserwirtschaftliche Grundsatzplanungen der Bundeswasserbauverwaltung und wurden in Österreich für eine Vielzahl von größeren Flüssen erstellt. Das Hauptziel eines Gewässerbetreuungskonzeptes liegt im Hochwasserschutz des Menschen und der Siedlungen unter gleichzeitiger Erhaltung oder Verbesserung der ökologischen Funktionsfähigkeit. Im speziellen Fall der Traisen ist zudem die Sicherung des Grundwasserhaushalts ein Hauptanliegen. Das „GBK Traisen-Wilhelmsburg bis Donau“ wurde zwischen 1995 und 1999 erstellt und setzte sich aus mehreren Arbeitsphasen zusammen. Nach einer Vorstudie erfolgten eine umfassende Aufnahme des Istzustands und die Ausarbeitung eines gewässerspezifischen Leitbildes. Aus der Defizitanalyse zwischen Istzustand und Leitbild leitete sich das Maßnahmenkonzept ab.

Der Schwerpunkt des GBK TraisenWilhelmsburg bis Donau lag auf dem Gewässerabschnitt flussauf der Mündungsstrecke, der im Zuständigkeitsbereich der Bundeswasserbauverwaltung liegt. Da eine funktionierende Vernetzung zwischen Donau und Traisen essenziell für die ökologischen Verhält- 
nisse in der Traisen bis St. Pölten ist, befasste sich das Projektteam auch mit dem Mündungsabschnitt, dessen Instandhaltung als Anlagenbestandteil des Donaukraftwerkes Altenwörth der VHP obliegt (Eberstaller et al. 1999). Die Bestandsaufnahme zeigte hier den unbefriedigenden ökologischen $\mathrm{Zu}$ stand. Wegen der Lage außerhalb von Siedlungsgebieten wurde das Potenzial für Verbesserungen im Mündungsabschnitt als besonders hoch erachtet. Es wurden schließlich vier generelle Maßnahmentypen einer Umgestaltung formuliert:

- Die Laufverlegung mit mäandrierender Linienführung, um die Habitatvielfalt zu verbessern,

- die Wiederherstellung des Längskontinuums durch die Verlegung des Traisenlaufs in das sogenannte Weingartlwasser,

- die Absenkung des Umlands zur Schaffung von Weichholz-Austandorten und

- die Vernetzung von Traisen und ausgewählten Nebengewässern der Traisen-Donau-Auen. Gewässer mit wertvollen Tier- oder Pflanzenarten, die keine regelmäßige Anbindung vertragen, sollten dabei gezielt ausgespart werden.

Um die Finanzierbarkeit eines derartigen Vorhabens zu sichern, wurde bereits im GBK vorgeschlagen, die Wiederherstellung von Weichholzauen mit einer Verwertung des anfallenden Kiesmaterials $\mathrm{zu}$ kombinieren. Es wurde jedoch darauf hingewiesen, dass diese Materialentnahme nur über große Flächen, vergleichsweise geringe Tiefen und nach ökologischen Kriterien erfolgen kann und sich damit wesentlich vom herkömmlichen, flächig konzentrierten Schotterabbau unterscheidet. Zur weiteren, detaillierten Abklärung einer ökologischen Mündungsumgestaltung wurde eine Machbarkeitsstudie vorgeschlagen.

Insgesamt gesehen war zum damaligen Zeitpunkt die Idee zum Rückbau des Traisen-Unterlaufs und des Mündungsbereiches freilich nicht mehr als eine schöne Vision.

\section{Die Fortsetzung der Visionen bis 2004}

Nach der Fertigstellung des GBK war eine Realisierung des großräumigen Flussbauprojekts undenkbar. Neben fehlender Finanzierbarkeit mangelte es damals am Interesse von VHP für ökologisch notwendige Veränderungen im Traisen-Unterlauf sowie an der Mitwirkung der Grundeigentümer. Für ein derartiges Vorhaben fehlten zum damaligen Zeitpunkt auch noch ausreichende gesetzliche Rahmenbedingungen und Fördermöglichkeiten.

In den Jahren um die Jahrtausendwende gab es trotzdem immer wieder informelle Hintergrundgespräche zu der nie ganz in Vergessenheit geratenen Vision. Im Herbst 2003 schließlich beschlossen die Vertreter der drei größeren Grundeigentümer im Gebiet (Forstverwaltung Grafenegg, $\mathrm{Au}$ gustiner-Chorherren-Stift Herzogenburg und Agrargemeinschaft Lehenteil) einen neuerlichen Anlauf für eine Realisierung der Neugestaltung der unteren Traisen samt Mündung und nahmen Kontakt zum Institut für Hydrobiologie und Gewässermanagement (IHG) der Universität für Bodenkultur Wien (BOKU) auf. Das hier tätige wissenschaftliche Team erarbeitete rasch erste Projektskizzen. Sie umfassten einen möglichen neuen Gewässerverlauf der Traisen sowie eine Grobabschätzung der bei Umsetzung des Projektes anfallenden Erd- und Kiesmassen.

Im März 2004 wurde durch das Projektteam „Rückbau Untere Traisen“ die erste konkrete Information zur Projektidee beim Amt der NÖ Landesregierung, Gruppe Wasser, vorgestellt und eine mögliche Machbarkeitsstudie erörtert. Wichtige Themen waren in diesem Zusammenhang auch Abstimmungen über die Projektträgerschaft, Kosten/ Finanzierung einer Vorstudie etc.

Seitens der Gruppe Wasser wurde klargestellt, dass das Land Niederösterreich nicht Hauptinteressent dieses Projektes sei und deshalb auch nicht federführend die Projektträgerschaft übernehmen könne. Zunächst herrschte die Meinung vor, dass das Hauptinteresse dieses Projektes bei den betroffenen Grundeigentümern liegen würde und diese deswegen auch die Projektträgerschaft übernehmen müssten. Aufgrund der Auffassungsunterschiede kam man überein, dass eine Machbarkeitsstudie beauftragt werden soll, um nach deren Vorliegen die Interessenslagen deutlicher abgrenzen, gewichten und im Anschluss die Frage der Projektträgerschaft auf fundierter Basis diskutieren zu können.

Das damalige Bundesministerium für Land- und Forstwirtschaft, Umwelt und Wasserwirtschaft (BMLFUW) brachte in weiterer Folge die Bereitschaft zur Teilnahme und Mitarbeit am Projekt zum Ausdruck. Das BMLFUW wies darauf hin, dass unter den zwischenzeitlich geänderten gesetzlichen Rahmenbedingungen - speziell in Hinblick auf die Umsetzung der EU-Wasserrahmenrichtlinie (EU-WRRL) - zukünftig auch geänderte Anforderungen an die Instandhaltung zu berücksichtigen seien. VHP stand der Projektidee zwar weiterhin kritisch gegenüber, die Erkenntnis, dass eine Umsetzung des Projekts ohne Mitwirkung des Kraftwerksbetreibers nicht möglich sein werde, führte aber schließlich auch zur intensiven Auseinandersetzung von VHP mit den Projektzielen.

Als zentrales Ziel der Machbarkeitsstudie wurde festgelegt, dass sämtliche projektbedingte Vor- und Nachteile für alle Beteiligten (Grundeigentümer, VHP, Fischerei etc.) zu erfassen, zu bewerten und die Auswirkungen auf alle berührten Rechte abzuschätzen sind. Der Vertreter des Stiftes Herzogenburg übernahm ehrenamtlich die Koordination der Projektgruppe. Die NÖ Landesregierung erklärte sich bereit, organisatorisch Hilfe zu leisten und Abstimmungen mit VHP und Wasserstraßendirektion (WSD; heute via donau) als mögliche Projektpartner zu übernehmen.

\section{Die Machbarkeitsstudie 2005}

Im Juni 2004 wurden das Institut für Hydrobiologie und Gewässermanagement der Universität für Bodenkultur Wien, die DonauConsult Zottl \& Erber ZT-GmbH und ezb -TB Eberstaller beauftragt, die wirtschaftliche und technische Machbarkeit einer umfassenden Revitalisierung des Traisen-Unterlaufs und der Traisen-Mündung unter Berücksichtigung einer Kiesverwertung zu untersuchen. Einen wichtigen Hintergrund für das Revitalisierungsvorhaben stellten zu diesem Zeitpunkt bereits die 2003 in nationales Recht integrierten Ziele der EU-Wasserrahmenrichtlinie dar. Diese fordern explizit die Verbesserung des ökologischen Zustandes von Gewässern. Die Umgestaltung des Traisen-Unterlaufs wurde als eine wesentliche Chance zur Verbesserung der ökologischen Verhältnisse im unmittelbaren Projektgebiet angesehen. Darüber hinaus waren positive Effekte auf die flussauf gelegene Traisen und die Donau in der Stauwurzel des KW Greifenstein zu erwarten. 


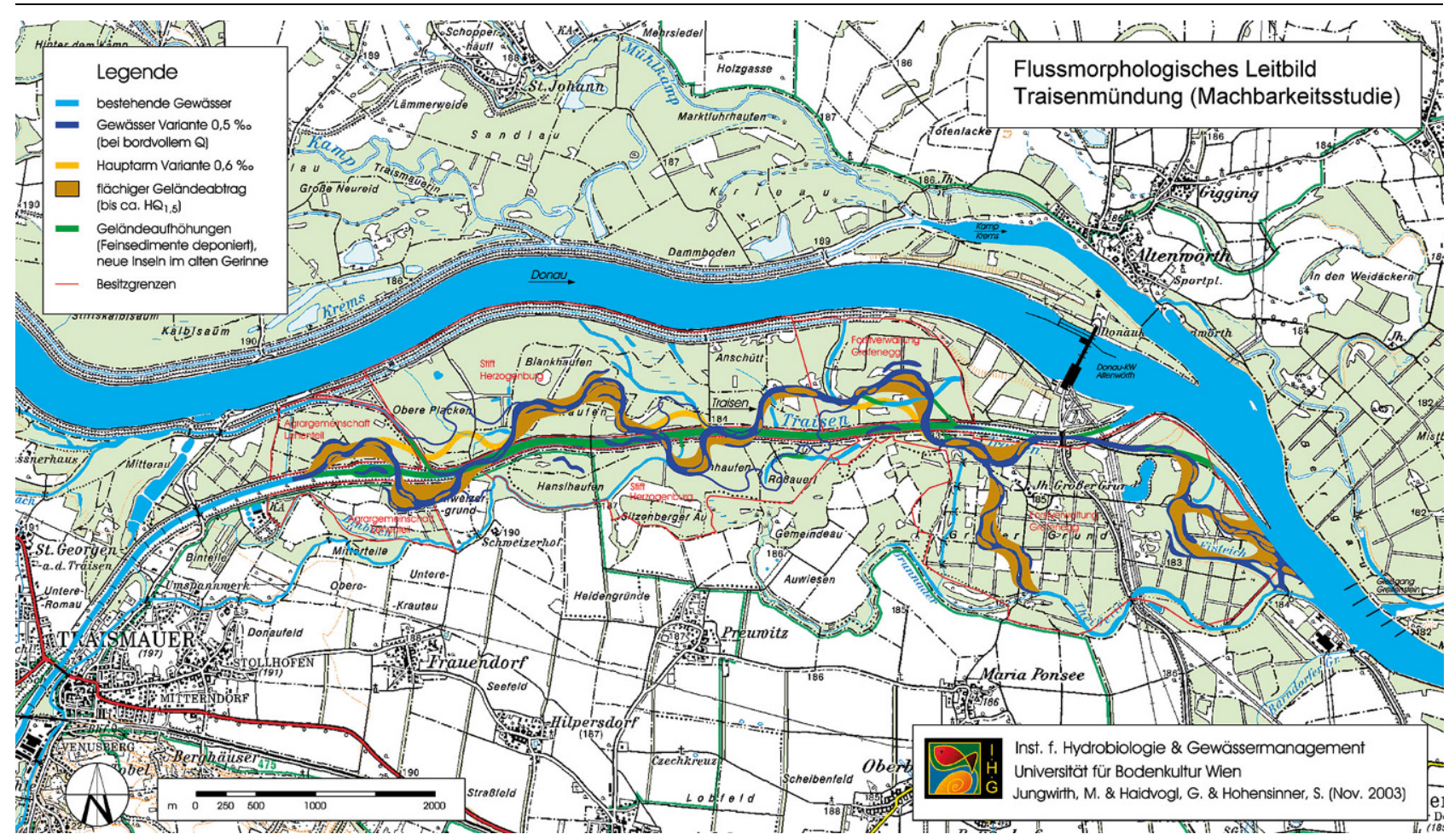

Abb. 1 Schematische Darstellung des morphologischen Referenzzustands für den künftigen Traisen-Unterlauf mit angebundenen Altarmen und Auweihern

In den Jahren 2004 und 2005 wurde die Ausarbeitung zur Neugestaltung der Traisen im Mündungsbereich und der umgebenden Donauauen weitergeführt. Die Studie wurde durch die Grundeigentümer (Forstverwaltung Grafenegg, Augustiner-Chorherren-Stift Herzogenburg und Agrargemeinschaft Lehenteil) sowie BMLFUW, Land NÖ (Bundeswasserbauverwaltung) und den NÖ Landesfischereiverband finanziert.

Ziel der Machbarkeitsstudie (Jungwirth et al. 2005) war es, zu prüfen, ob die Herstellung einer ökologisch vielfältigen, naturnahen neuen Traisen-Mündungsstrecke bei den umfassenden technischen (z.B. Einfluss auf bestehende Infrastruktur und Schifffahrtsanlagen), wasserwirtschaftlichen (v. a. Hochwasserschutz, Grundwasser) und ökonomischen (Finanzierbarkeit) Rahmenbedingungen möglich ist. Die Grundlagen zum Hochwasserschutz, den Grundwasserverhältnissen und zum ökologischen Zustand wurden zum größten Teil auf Basis des GBK Traisen (publiziert 1999) und aus Informationen von Ämtern und Behörden zusammengestellt. Basierend auf diesen Unterlagen wurde auch ein flussmorphologisches Leitbild entwickelt, das Lage, Gefälleverhältnisse und Bodenaufbau (Donaualluvion) in den ehemaligen Donauauen berücksichtigte (siehe Abb. 1).

Die Erhebungen und Analysen konzentrierten sich in der Machbarkeitsstudie vor allem auf den Untergrundaufbau sowie eine verlässlichere Abschätzung des Kies- und Feinsedimenthorizonts und der zu erwartenden Aushubkubatur, die zur Finanzierung des Projekts beitragen sollte.

In die Abstimmung eines potenziellen Korridors (=Grenze der Baueingriffe) für den zukünftigen TraisenUnterlauf waren relevante Behörden, Verwaltungsstellen und Interessenten einbezogen. Die in Kooperation mit der Abteilung Naturschutz des Amtes der Niederösterreichischen Landesregierung konzipierten Maßnahmen sollten den Erhalt bestehender Schutzgüter und Lebensräume im Gebiet sicherstellen sowie in der FFH-Richtlinie gelistete Lebensraumtypen schaffen und Arten fördern. Im Vordergrund stand die Initiierung neuer Weichholzauwälder. Im Zuge der Planung des Korridors mussten der Hochwasserschutz für die umliegenden Gemeinden sowie der Grundwasserspiegel beachtet werden. Die in der Machbarkeitsstudie ebenfalls angesprochene Logistik der Kiesentnahme und -verbringung sah einen Materialabtransport per Lkw vor. Da der jährliche Kiesaushub und dessen Verfuhr in einem angenommenen wirtschaftlich vertretbaren Transportradius von $50 \mathrm{~km}$ begrenzt war, wurde eine entsprechend lange Bauzeit (10 Jahre) als Kalkulationsbasis gewählt. Auf die eventuell höheren Kosten im Falle eines Schiffstransports auf der Wasserstraße Donau wurde bereits allgemein hingewiesen.

Basierend auf all diesen Überlegungen und Anforderungen wurde schließlich ein 12,5 km langer „Planungskorridor" festgelegt und in 4 Abschnitte untergliedert. In mehreren Varianten wurden Szenarien untersucht und kostenmäßig evaluiert. Schließlich wurde die sogenannte „Variante III“ als ökologisch, wasserwirtschaftlich, technisch und wirtschaftlich optimierte Variante empfohlen (siehe Abb. 2).

Für diese Variante wurden unter den damaligen Rahmenbedingungen Gesamtkosten von rund 13 Mio. $€$ für Planung, Verfahren, Bau, Entschädigungen und Aufforstungen ermittelt. Nach Abzug eines erwarteten Kieserlöses von rund 6 Mio. $€$ blieb ein Finanzierungsbedarf von etwa 7 Mio. $€$. 


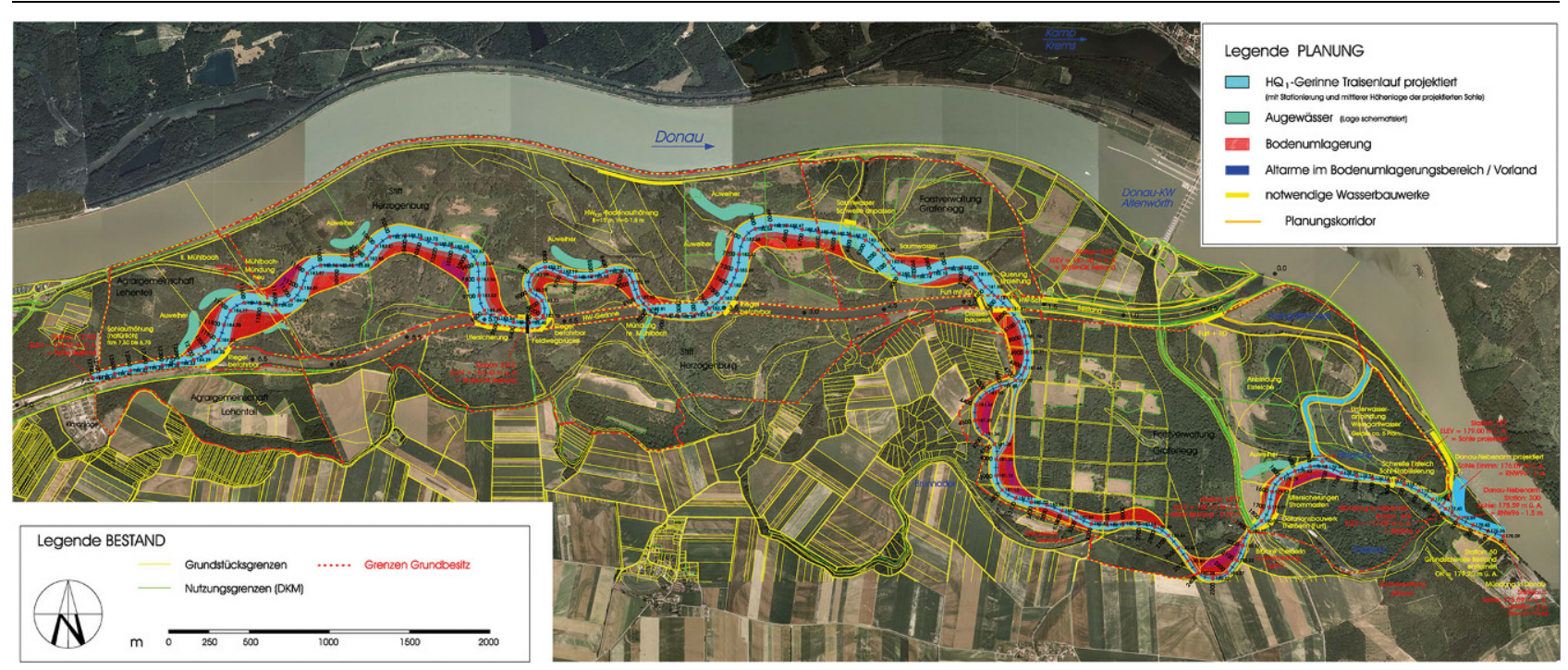

Abb. 2 In der Machbarkeitsstudie 2005 vorgeschlagenen Projektvariante für einen naturnahen Traisen-Unterlauf

\section{Der erste LIFE-Antrag 2005}

Als wesentlicher Beitrag zur Abdeckung des verbleibenden Finanzierungsbedarfes wurde eine Förderung aus dem LIFE-Programm der Europäischen Union gesehen, da bereits andere große Flussbauprojekte in Niederösterreich in den Jahren 1999 bis 2004 mit einer derartigen Förderung umgesetzt werden konnten. Neben dem LIFE-Projekt „Huchen“ war dies vor allem das LIFEProjekt „Wachau“, in dem große Donaunebenarme in der Wachau geschaffen wurden. Aufgrund der einschlägigen Erfahrung mit der erfolgreichen Antragstellung wurde das Büro Freiwasser zur Unterstützung beigezogen.

Die Umwelt-Förderung aus dem LIFE-Programm wird in einem EUweiten Wettbewerb vergeben. Förderwürdige Projekte müssen den Zielen des LIFE-Programms entsprechen und innovativ sein. Der Unterlauf der Traisen liegt im Natura-2000-Gebiet Nr. 16 „Tullnerfelder Donauauen“, dem größten zusammenhängenden Augebiet Österreichs. Somit war eine wesentliche Voraussetzung für die Förderung über die damalige Schiene „LIFE-nature 2006“" gegeben.

Als Projektträger konnte VHP gewonnen werden. Durch die Teilnahme am Projekt erwartete sich das Unternehmen, dass Anforderungen aus der Umsetzung der Wasserrahmenrichtlinie in einem zukunftsweisenden, ökologisch hochwertigen Umweltprojekt bei hoher Kosteneffizienz umgesetzt werden können.
Die ökologischen Ziele für den unmittelbaren Gewässerlauf waren eine wesentliche Grundlage für eine potenziell erfolgreiche LIFE-Antragstellung. Ein durchgängiges, variables Flussbett mit hoher Lebensraumvielfalt und intakter Uferverzahnung und Dynamik war geeignet, die Bestände der laut FFH-Richtlinie geschützten Fisch-, Libellen- und teils auch Amphibienarten zu sichern bzw. zu verbessern. Das wichtigste und erfolgversprechendste Argument für die LIFE-Förderung lag aber in den geplanten, häufig überstauten flachen Uferzonen. Auf ihnen kann sich die zum Fluss gehörende „Weiche $A u$ “ selbstständig entwickeln. Diese tiefliegenden Bereiche sollten laut Projektidee baulich angelegt und der Gehölzsukzession überlassen oder autochthon bepflanzt werden. Gerade die „Weiche Au“ aber ist ein prioritärer FFH-Lebensraumtyp (Bezeichnung „91E0*“), also besonders wertvoll. Wie auch anderswo in Europa wurden die Bedingungen für die Entwicklung dieses Auwald-Lebensraumes durch Regulierung und Kraftwerksbau an Flüssen stark dezimiert. Zusätzlich zu einem $12,5 \mathrm{~km}$ langen, neuen Fluss waren 82 ha neu entstehender hochwertiger Auwald ein weiterer wesentlicher Beitrag zur Verbesserung des Auenlebensraumes.

Der erste Antrag auf eine LIFEFörderung wurde am 30. September 2005 bei der Abteilung Naturschutz des Amtes der Niederösterreichischen Landesregierung zur Weiterleitung an die EU Kommission eingebracht. Im Antrag wurden die unterschiedlichen ökologischen, wasserwirtschaftlichen und technisch nötigen Maßnahmen innerhalb des Planungskorridors dargestellt. Der Planungskorridor wurde im Vergleich zur Machbarkeitsstudie aufgrund diverser technischer Überlegungen und der Berücksichtigung von Zwangspunkten weiter optimiert. Die biologisch-ökologischen Schutzüberlegungen für bestehende Habitate oder Vorkommen von geschützten Tier- und Pflanzenarten wurden im Detail beschrieben. Das Wissen darüber sollte später durch Erhebungen im Zuge der Umweltverträglichkeitserklärung (UVE) noch zunehmen und zu weiteren Adaptierungen des Korridors führen.

Die eingereichte Projektfassung erforderte nach Abzug der Gegenfinanzierung aus dem Kieserlös Projektkosten von insgesamt 5,5 Mio. $€$ netto. Davon sollte die EU aus LIFE-Mitteln $60 \%$ (3,3 Mio. €), VHP 1,1 Mio. $€$ und die Partner und Kofinanzierer (Niederösterreichische Bundeswasserbauverwaltung, Niederösterreichischer Fischereiverband sowie Fischereirevierverbände, Landschaftsfonds Niederösterreich und via donau) in Summe 1,1 Mio. $€$, zusammen also die restlichen $40 \%$, beisteuern. Für die Fischereiverbände war das die größte bis dahin je beschlossene Förderung. Ein Jahr später, am 5. Mai 2006, lehnte die EU-Kommission die Projektförderung jedoch ab.

\section{Der zweite LIFE-Anlauf 2007}

Im Jahr 2007 wurde für die LIFE-Programmphase „LIFE+2007-2013“ ein neuer Antrag für den Schwerpunkt 
„LIFE+ Nature \& Biodiversity“ erstellt. Die von der EU-Kommission angemerkten und weiter unten näher erläuterten Defizite des ersten LIFE-Antrags wurden bestmöglich eliminiert. Als Projektlaufzeit wurde 2009 bis 2014 angesetzt.

Die überarbeitete Projektfassung erforderte nach Abzug der Gegenfinanzierung aus dem erwarteten Kieserlös (5,3 Mio. €) Projektkosten von insgesamt ca. 7,5 Mio. $€$ netto. Davon sollten die Europäische Union aus LIFEMitteln ca. $70 \%$ (5,3 Mio. €), die VHP 1,1 Mio. $€$ und die Partner und Kofinanzierer in Summe 1,1 Mio. $€$, also die restlichen $30 \%$, beisteuern. Als Partner und Kofinanzierer konnten wieder jene Institutionen genannt werden, die auch beim ersten Antrag dabei waren.

Da die Berechnung nach Erfahrungswerten bei der Ablehnung des ersten LIFE-Antrages als nicht ausreichend eingestuft worden war, wurden die Entschädigungsansprüche der Grundeigentümer nun auf Grundlage eines unabhängigen Gutachtens der Landwirtschaftskammer nachvollziehbar bewertet.

Des Weiteren bestätigte VHP, dass sie eine Verpflichtung zum Abtransport des Kiesaushubs per Schiff über die Wasserstraße Donau als Bedingung in die Bauausschreibung aufnehmen würde. $\mathrm{Zu}$ diesem Zeitpunkt war aus den Gesprächen mit Vertretern der Standortgemeinden bereits erkennbar, dass mit erheblichem Widerstand gegen einen Transport auf der Straße und durch die Ortschaften zu rechnen sein würde, der sich auch in einem potenziellen UVPVerfahren artikulieren würde. Die Beschränkung auf den Abtransport per Schiff erwies sich im weiteren Projektverlauf jedoch als erhebliche und projektgefährdende Belastung, da - wegen der großen Transportweiten und zusätzlichen Umschläge - offenbar nur wenige am Markt verfügbare Verwerter in der Lage sind, den auf Schiffen transportierten Kies mit wirtschaftlich vertretbarem Aufwand einer geeigneten Nutzung zuzuführen.

Ein weiterer Kritikpunkt betraf die Darstellung der Baukosten und der Kosten des Planungsprozesses sowie der Projektphase bis zur Erlangung der behördlichen Bewilligungen, die daher im neuen Antrag detailliert wurden.

Anfang Juni 2008 sandte die EUKommission eine Rückmeldung mit insgesamt 21 zum Teil sehr komplexen und detaillierten Fragen und räumte einen 14-tägigen Zeitraum zur Beant- wortung ein. Das bedeutete einen sehr hohen Einsatz aller Beteiligten, mussten doch auch neue Grafiken, GIS-Auswertungen, Evaluierungen etc. angefertigt und Bestätigungen unterschrieben werden. Eine wesentliche Bedingung der EU-Kommission für die Zusage von 5,2 Mio. $€$ war, dass die Darstellung des Projektbudgets neben den Kosten der Kiesentnahme auch den geschätzten Erlös umfassen sollte. Der Kieserlös musste somit als Eigenmittel von VHP in die Projektkosten eingerechnet werden. Für die Bestätigung über VHPEigenmittel in der Höhe von 6,4 Mio. $€$ statt zuvor 1,1 Mio. $€$ war aus formalen Gründen ein Beschluss des Aufsichtsrates notwendig, dessen nächste Sitzung jedoch erst für Herbst angesetzt war. Nur eine eigens aufgesetzte Erklärung, Telefonate mit einem EU-Kommissionsmitglied und ein Formular mit der Verpflichtung zur Nachbringung konnten den Genehmigungsprozess am Laufen halten.

Nachdem die EU-Kommission die Antworten und detaillierten Ausführungen akzeptiert und das LIFE+ Komitee das Projekt Ende Juli genehmigt hatte, erging am 14. August 2008 schriftlich die Aufforderung, alle Änderungen einzuarbeiten und bis Ende September 2008 den aktualisierten Antrag erneut zu übermitteln. Für den sogenannten "revised proposal“ mussten wieder alle Finanzzusagen der Partner und Ko-

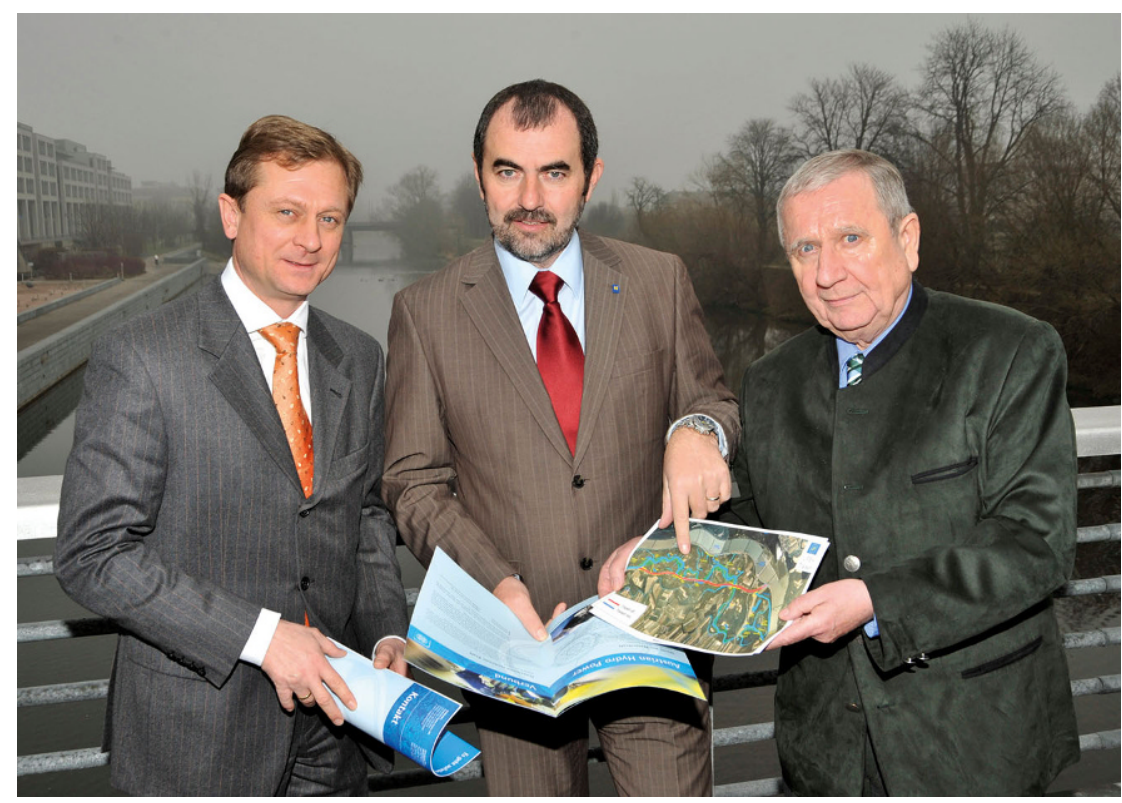

Abb. 3 Pressefoto Dezember 2008 anlässlich der Pressekonferenz im Landhaus St. Pölten finanzierer mit Unterschrift erneuert werden!

Über die LIFE-Förderung wurde ein Vertrag abgeschlossen, dessen Grundlagen das akzeptierte Projekt inklusive Finanzzusagen aller Beteiligten sowie die Vertragsbestimmungen der EU bilden. Nach der Unterzeichnung durch VHP und Übermittlung an die Kommission im Dezember 2008 stand dem offiziellen Projektstart mit Anfang 2009 nichts mehr im Wege.

\section{Der Start des LIFE+ Projekts 2009}

Bereits unmittelbar nach Förderzusage der EU-Kommission erfolgte die Implementierung der Projektleitung und Projektbuchhaltung bei VHP. Ein Team von externen Büros (Donauconsult, ezb-TB Eberstaller, Büro freiwasser und Institut für Hydrobiologie und Gewässermanagement an der Universität für Bodenkultur Wien) wurde mit der Fortführung der Projektierung beauftragt. Die Stakeholder wurden über Projektstart und Inhalte informiert. Im Dezember 2008 erfolgte die Präsentation des Vorhabens „LIFE+ Traisen“ im Rahmen einer Presskonferenz im Landhaus St. Pölten (Abb. 3).

Bereits im Februar 2009 finalisierte das Projektteam ein Vorprojekt, das eine Zusammenstellung aller zum damaligen Zeitpunkt entwickelten Planungsarbeiten und deren Grundlagen 
enthielt. Damit war einer der wichtigsten Schritte zur Darstellung des Vorhabens in Form einer Präsentations- bzw. Diskussionsgrundlage bei den Stakeholdern und Behörden gemacht. Im Vorprojekt fanden sich auch Informationen zu Grundwasser- und Hochwassersituation; aktuellere biologische Detailinformationen zum Projektgebiet waren damals noch wenig detailliert.

\section{Das UVP-Verfahren 2009 bis 2012}

Das Vorprojekt diente insbesondere auch als Grundlage des UVP-Feststellungsantrages, den VHP 2009 bei der zuständigen UVP-Behörde des Landes Niederösterreich einbrachte. Mit Bescheid vom 20. April 2009 stellte die NÖ Landesregierung erwartungsgemäß fest, dass die für das Vorhaben erforderlichen Rodungen den Tatbestand des Anhangs 1, Z 46a UVP-G 2000 (Rodungen auf einer Fläche von mindestens 20ha) erfüllen. Nach Berufung durch zwei Standortgemeinden stellte der Umweltsenat mit Bescheid vom 25. August 2009 darüber hinaus fest, dass auch der Tatbestand des Anhangs 1 Z 25a UVP-G 2000 (Entnahme von mineralischen Rohstoffen im Tagbau ... mit einer Fläche von mindestens 20ha) verwirklicht wurde, und die Umweltverträglichkeitsprüfung daher nicht im vereinfachten, sondern im ordentlichen Verfahren durchzuführen war.

In Anbetracht des in Anhangs 1 Z 41 UVP-G 2000 (Anlegung oder Verlegung von Fließgewässern) normierten Ausnahmetatbestandes für „Maßnahmen zur Verbesserung der ökologischen Funktionsfähigkeit der Gewässer (Renaturierungen)" bestehen aus Sicht der Verfasser begründete Zweifel, ob die Durchführung einer Umweltverträglichkeitsprüfung bei Vorhaben wie LIFE+ Traisen tatsächlich der Intention des Gesetzgebers entspricht. Es ist jedenfalls offenkundig, dass die durch Einzelmaßnahmen (Rodung, Kiesentnahme) begründete UVP-Pflicht aufgrund des damit verbundenen deutlichen zeitlichen und finanziellen Mehraufwands eine erhebliche Hürde für die Umsetzung weiterer großer Gewässerrevitalisierungsprojekte darstellt und damit einen wohl unerwünschten Lenkungseffekt ausübt.

Die wasserbauliche Einreichplanung sowie die Erstellung der erforderlichen Unterlagen für die Umweltverträglichkeitserklärung (UVE) nahmen rund ein Jahr in Anspruch. Der sehr aufwendige, interdisziplinäre Planungsprozess wurde in regelmäßigen Diskussionen des Projektteams koordiniert.

Die zusammenfassende Bewertung der Projektauswirkungen auf das Schutz gut Pflanzen, Tiere und deren Lebensräume im Rahmen der UVE basierte auf neuen Erhebungen zu verschiedenen aquatischen und semiaquatischen Tiergruppen (Makrozoo- und Phytobenthos, Fische, Libellen, Amphibien) sowie $\mathrm{zu}$ ausgewählten terrestrischen Tiergruppen (Käfer, Schmetterlinge, Heu- und Fangschrecken, Fledermäuse, Vögel). Zudem wurden die Vegetation des Projektgebiets und die Hydromorphologie der Traisen berücksichtigt. Bei den Erhebungen der Indikatorgruppen wurden insgesamt 225 Tierarten bzw. Lebensraumtypen nachgewiesen. Für weitere 24 Tierarten wurde von einem Vorkommen im Projektgebiet ausgegangen, da sie in den angrenzenden Regionen des Natura-2000-Gebiets „Tullnerfelder Donauauen“ vorkommen und im Projektgebiet prinzipiell Lebensräume vorfinden würden. Insgesamt waren 118 Arten oder ca. 50\% in der Roten Liste erfasst, wobei alle Gefährdungsstufen bis hin zu „vom Aussterben bedroht" vertreten waren. In der UVE wurde systematisch bewertet, wie die vorgefundenen Arten bzw. Vegetationstypen auf einzelne Maßnahmen und Aktivitäten während Bau und Bestand reagieren würden. Im Fall einer $\mathrm{zu}$ erwartenden Verschlechterung der Bestände durch das Projekt, vor allem von geschützten Tier- und Pflanzenarten, hätten geeignete Gegenmaßnahmen geplant werden müssen.

Die beiden höchsten Sensibilitätsstufen „sehr hoch sensibel“ und „hoch sensibel“ wurden vor allem einzelnen Lebensraumtypen, Vögeln, Amphibien, Libellen, Schmetterlingen und Fledermäusen zuerkannt.

Im Zuge der UVE-Erstellung bzw. der parallel laufenden wasserbaulichen Einreichplanung wurden Verlauf und Detailgestaltung des neuen Traisenkorridors in zahlreichen Projektbesprechungen iterativ optimiert, um die nachgewiesenen Schutzgüter sowohl während der Bauzeit als auch im endgültigen Zustand zu sichern (vgl. Artikel „LIFE+ Traisen: Der neue Fluss - die Umsetzung“; Eberstaller et al. 2018).

Am 14. Oktober 2010 wurde schließlich bei der NÖ Landesregierung die Genehmigung des Vorhabens „LIFE+ Lebensraum im Mündungsabschnitt des Flusses Traisen“ beantragt. Auf- grund der Ergebnisse der Vollständigkeitsprüfung durch die UVP-Behörde des Landes Niederösterreich und ihrer Gutachter wurde am 19. Jänner 2011 ein Verbesserungsauftrag erteilt. Nach Ergänzung der Unterlagen im März und Mai 2011 wurden die Projektunterlagen im Juni 2011 öffentlich aufgelegt. Vorbereitend erfolgten bereits Mitte Mai Bürgerinformationsveranstaltungen in Zwentendorf, Traismauer und Altenwörth. Im Rahmen von Informationsnachmittagen während der öffentlichen Auflage wurden in den Gemeinden Fragen zum Vorhaben beantwortet.

Aufgrund der im Juli 2011 erhobenen Einwendungen mehrerer Beteiligter wurde die wegen der Umgestaltung erforderliche Neutrassierung des Donauradweges im Mündungsbereich adaptiert. Auf Grundlage des Umweltverträglichkeitsgutachtens vom Oktober 2011 wurde das Vorhaben im Zuge einer mündlichen Verhandlung am 23. November 2011 erörtert. Nach der Verhandlung wurden noch einige Unterlagen bis 30. November 2011 ergänzt. Das UVP-Verfahren wurde schließlich mit positivem Bescheid der NÖ Landesregierung vom 24. April 2012 abgeschlossen.

Etwa zur gleichen Zeit erhielt LIFE+ Traisen den Neptun Wasserpreis 2011 als bestes Wasserprojekt Österreichs. Die Jury begründete die Auszeichnung damit, dass die Herausforderung, den Schutz der Gewässer und die Sicherung naturnaher Lebensräume mit den Erfordernissen des Hochwasserschutzes und der Wasserkraftnutzung in Einklang zu bringen, in diesem Projekt vorbildlich gelöst wurde.

\section{Die Projektänderung 2012}

Im Gegensatz zu den optimistischen Erwartungen der Vorphasen zeigten die Ergebnisse des Vergabeverfahrens für die Bauleistungen klar, dass die Möglichkeiten zur wirtschaftlichen Verwertung des anfallenden Aushubmaterials stark eingeschränkt waren und auch die Umsetzung des gesamten Vorhabens gemäß dem der UVP zugrunde liegenden Zeitplan bis Ende 2015 nicht möglich war. Es wurden daher drei Schritte gesetzt:

- Umsetzungszeitraum: Nach einem Fristerstreckungsansuchen verlängerte der Landeshauptmann mit Bescheid vom 17. Mai 2013 die Bauvollendungsfristen bis 31. Dezember 2019. Zusätzlich zu den nationalen 


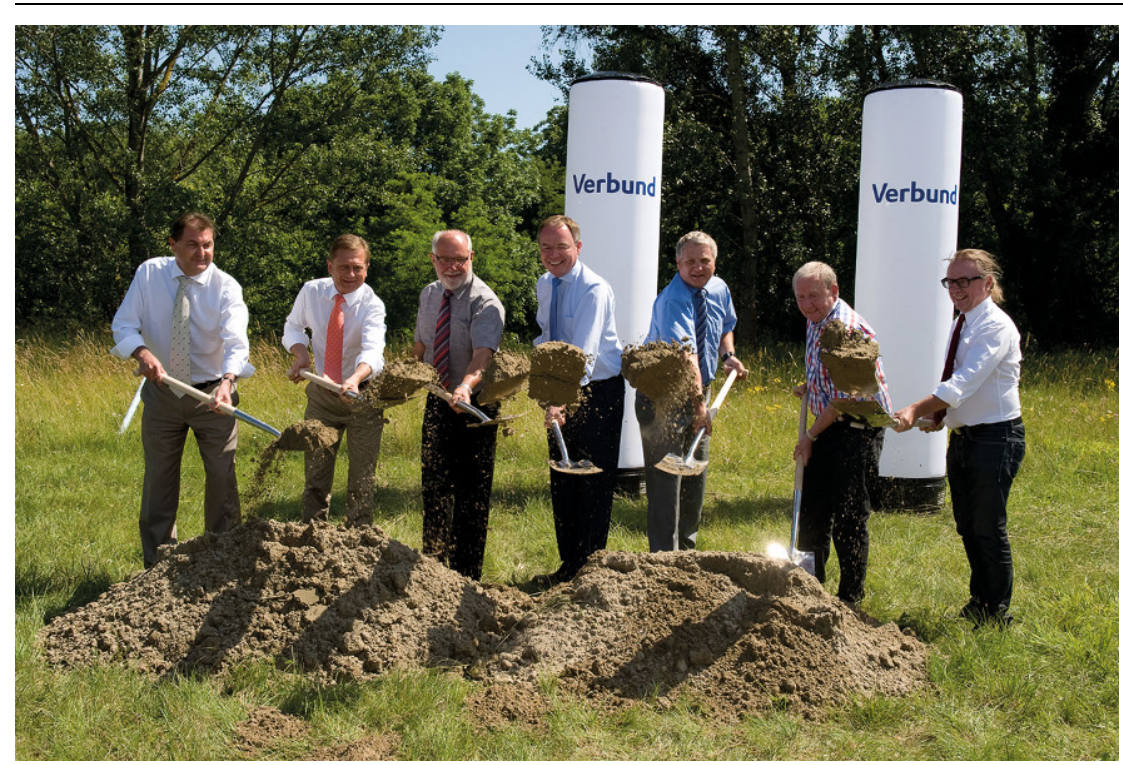

Abb. 4 Pressefoto Spatenstich 1. Juli 2013

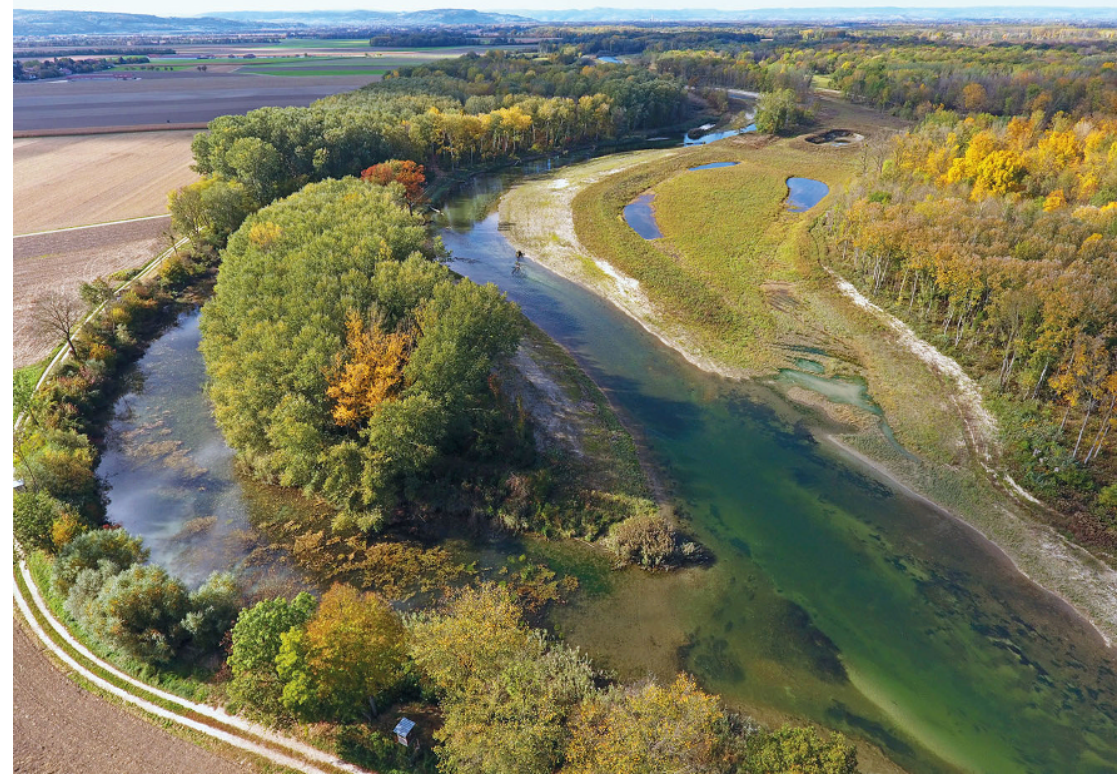

Abb. 5 Zur Veranschaulichung: Ein kleiner Ausschnitt der neuen 9,5 km langen Flusslandschaft ein Jahr nach Baufertigstellung

Änderungen musste auch der LIFE+ Vertrag inhaltlich und bezüglich des Zeitplans bis 2019 abgeändert werden.

- Maßnahmen: Im Rahmen einer aus Kostengründen notwendigen Redimensionierung des Vorhabens musste der westliche Bauabschnitt einge- geplanten Neuen Traisen verkürzte sich damit auf rund 9,5 km. In Hinblick auf die LIFE+ Förderung konnte der damit einhergehende Entfall von neu entstehendem Auenlebensraum durch Verbesserungsmaßnahmen für 30 ha Kalk-Halbtrockenrasen, eines weiteren prioritären $\mathrm{FFH}$ Lebensraumtyps im Au-Gebiet, kompensiert werden. Dies stellte einen weiteren Schritt $\mathrm{zu}$ einem integralen Flusslandschaftsprojekt dar. Die im Projektgebiet vorgesehene Errichtung von 11 Auweihern wurde zurückgestellt, später konnten zumindest vier Weiher realisiert werden.

- Kosten: Aufgrund der hohen Bedeutung des Vorhabens zur Erreichung des guten ökologischen Potenzials in der Donau erhöhte VHP ihren Beitrag zu den Projektkosten in erheblichem Ausmaß. Die Gesamtkosten des Projektes lagen schließlich bei 30 Mio. $€$, wovon VHP die Hälfte trug.

Diese deutlichen Adaptierungen erlaubten schließlich im Mai 2013 die Vergabe eines Bauauftrages. Der Spatenstich für LIFE+ Traisen erfolgte am 1. Juli 2013 (Abb. 4). Nach Abschluss der baulichen Umsetzung Ende 2016 ist die neue Traisen gut ins Augebiet eingebettet, die neue Flusslandschaft und ihre vielfältigen Habitate entwickeln sich nun selbständig weiter (Abb. 5). Diese Entwicklung wird bis zum Projektende mit diversen Monitoringarbeiten beobachtet und evaluiert.

Funding Open access funding provided by University of Natural Resources and Life Sciences Vienna (BOKU).

Open Access Dieser Artikel wird unter der Creative Commons Namensnennung 4.0 International Lizenz (http:// creativecommons.org/licenses/by/4. $0 /$ deed.de) veröffentlicht, welche die Nutzung, Vervielfältigung, Bearbeitung, Verbreitung und Wiedergabe in jeglichem Medium und Format erlaubt, sofern Sie den/die ursprünglichen Autor(en) und die Quelle ordnungsgemäß nennen, einen Link zur Creative Commons Lizenz beifügen und angeben, ob Änderungen vorgenommen wurden. • 
Eberstaller, J., Haidvogl, G., Jungwirth, M. (1999): Gewässerbetreuungskonzept Traisen. Kurzfassung. Bundesministerium für Land- und Forstwirtschaft und Amt d. N.Ö. Landesregierung, Abteilung WA3, Wien

Eberstaller, J., Schmalfuß, R., EberstallerFleischanderl, D., Gabriel, H., Kaufmann, T.,
Wimmer, H. \& Jungwirth, M. (2018): LIFE+

Traisen: Der neue Fluss - die Umsetzung, Österr. Wasser- und Abfallwirtschaft, Heft $5 / 6$

2018, Wien. https://doi.org/10.1007/s00506018-0474-8

Jungwirth, M., Haidvogl, G., Hohensinner, S.

Küblbäck, G., Schmalfuß, R., Eberstaller, J.
Pinka, P. (2005): Machbarkeitsstudie „Rückbau Traisenunterlauf und Traisenmündung“. Unveröffentlichter Endbericht, Wien 